\title{
Strontium Isotope Detection of Brine Contamination in the East Poplar Oil Field, Montana
}

Open-File Report 2010-1326 



\section{Strontium Isotope Detection of Brine Contamination in the East Poplar Oil Field, Montana}

By Zell E. Peterman, Joanna N. Thamke, Kiyoto Futa, and Thomas A. Oliver

In cooperation with the Fort Peck Tribes Office of Environmental Protection

Open-File Report 2010-1326 


\title{
U.S. Department of the Interior \\ KEN SALAZAR, Secretary \\ U.S. Geological Survey \\ Marcia K. McNutt, Director
}

\section{U.S. Geological Survey, Reston, Virginia: 2010}

\author{
For more information on the USGS — the Federal source for science about the Earth, its natural and living resources, \\ natural hazards, and the environment, visit http://www.usgs.gov or call 1-888-ASK-USGS \\ For an overview of USGS information products, including maps, imagery, and publications, \\ visit http://www.usgs.gov/pubprod \\ To order this and other USGS information products, visit http://store.usgs.gov
}

\begin{abstract}
Any use of trade, product, or firm names is for descriptive purposes only and does not imply endorsement by the U.S. Government.

Although this report is in the public domain, permission must be secured from the individual copyright owners to reproduce any copyrighted materials contained within this report.
\end{abstract}

Suggested citation:

Peterman, Z.E., Thamke, J.N., Futa, Kiyoto, and Oliver, T.A., 2010, Strontium isotope detection of brine contamination in the East Poplar oil field, Montana: U.S. Geological Survey Open-File Report 2010-1326, 20 p. 
Strontium Isotope Detection of Brine Contamination in the East
Poplar Oil Field, Montana

Zell E. Peterman, ${ }^{1}$ Joanna N. Thamke, ${ }^{2}$ Kiyoto Futa, ${ }^{1}$ and Thomas A. Oliver ${ }^{1}$

U.S. Geological Survey

1Denver, Colorado, ${ }^{2}$ Helena, Montana 


\section{Background}

- Brine contamination was documented in the East Poplar oil field as early as the mid-1980s by the U.S. Geological Survey (Levings, 1984; Thamke and Craigg, 1997; Thamke and Midtlyng, 2003).

- The City of Poplar's three public water-supply wells (COP-1, COP-2, and COP-3) are completed in the shallow aquifers downgradient from contaminated aquifers (S.S. Papadopulos \& Associates, Inc., 2008).

- Data from the North Dakota Department of Health in 1985 and Energy Laboratories, Inc., in 2009 indicate that chloride concentrations in water from COP-3 exceed the secondary maximum contaminant level of $250 \mathrm{mg} / \mathrm{L}$, as established by the U.S. Environmental Protection Agency (2010).

\section{₹USGS}




\section{Background (cont'd)}

- In April 2010, representatives from Federal, State, and city agencies; energy companies; and private consultants were invited to discuss investigations of chloride (Cl) concentrations and possible sources in groundwater near the City of Poplar.

- Sample design and approach were discussed in June 2010 by the above, and water samples were collected by the U.S. Geological Survey (USGS) during July 2010. 


\section{Approach}

- Groundwater samples were collected by the USGS Montana Water Science Center under the direction of Joanna Thamke and the Fort Peck Office of Environmental Protection under the direction of Christa Tyrrell.

- The analyses were conducted in the USGS Yucca Mountain Project Branch Geochemistry Laboratory in Denver, Colo.

- Strontium isotopes were measured by thermal ionization mass spectrometry (TIMS).

- Anion and cation concentrations were determined by ion chromatography (IC) and trace-metal concentrations by inductively coupled plasma-mass spectrometry (ICP-MS). 


\section{Locations of Sample Sites}

- Wells, Poplar River, and disposal sites that were sampled during July 2010 are labeled in map.

- Samples were analyzed for strontium isotopes, dissolved ions, and trace metals.

\section{₹USGS}

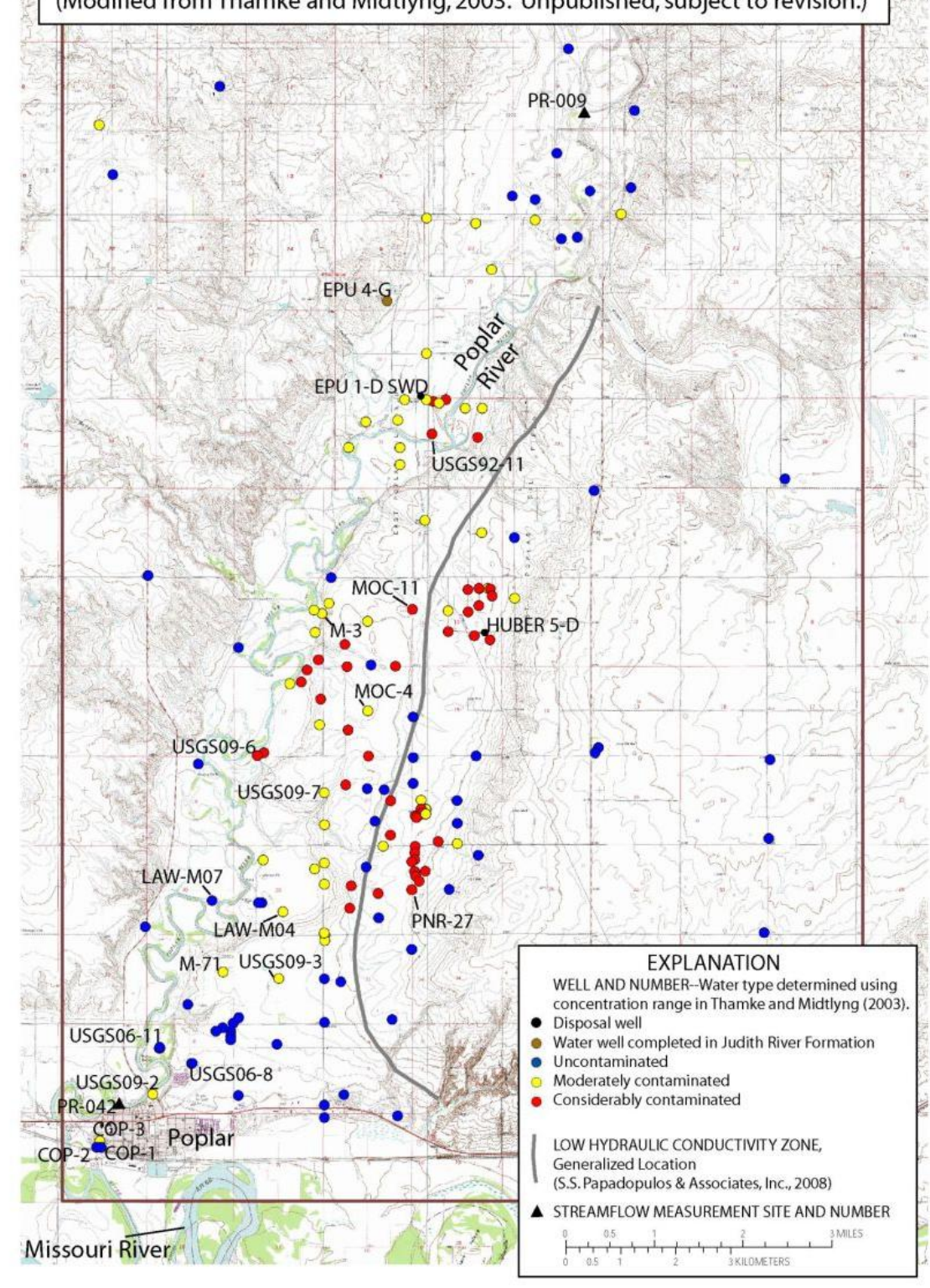

Open-File Report 2010-1326 


\section{Strontium Isotopes as Groundwater Tracers}

- Strontium (Sr) is an alkaline-earth element that closely follows calcium (Ca) in geochemical and hydrological cycles.

- Sr is composed of four isotopes: ${ }^{84} \mathrm{Sr},{ }^{86} \mathrm{Sr}$, ${ }^{87} \mathrm{Sr}$, and ${ }^{88} \mathrm{Sr}$, all of which are stable (nonradioactive).

- Fractionation of Sr isotopes in nature is extremely small and inconsequential in tracer studies.

- Some ${ }^{87} \mathrm{Sr}$ forms from the beta decay of ${ }^{87} \mathbf{R b}$ with a half-life of $\mathbf{4 8 . 8}$ billion years.

- Rocks with different ages and Rb/Sr ratios have different ${ }^{87} \mathrm{Sr} /{ }^{86} \mathrm{Sr}$ ratios. 


\section{Strontium Isotopes as Groundwater Tracers (cont'd)}

- ${ }^{87} \mathrm{Sr} /{ }^{86} \mathrm{Sr}$ ratios and $\mathrm{Sr}$ concentrations in groundwater are useful for detecting mixing of waters of different origins.

- Sr isotopes have been used near Goose Lake Waterfowl Protection Area to assess the impact of brine from oil-field operations on shallow groundwater within the Prairie Pothole Region (http:l/steppe_cr.usgs_gov/), and in the Weyburn field in Saskatchewan, Canada, to characterize the produced water from the Madison Group formations (Quattrocchi and others, 2006). 


\section{Mixing of Brines and Freshwater}

- Sr concentrations of freshwater and brine differ by two to three orders of magnitude.

- Mixing of waters with different ${ }^{87} \mathrm{Sr} /{ }^{86} \mathrm{Sr}$ ratios and reciprocal Sr concentrations will produce linear trends, from which end-member compositions and mixing proportions can be calculated (Faure, 1998). 


\section{${ }^{87} \mathrm{Sr} /{ }^{86} \mathrm{Sr}$ versus $1 / \mathrm{Sr}$ (Sr in $\mathrm{mg} / \mathrm{L}$ )}

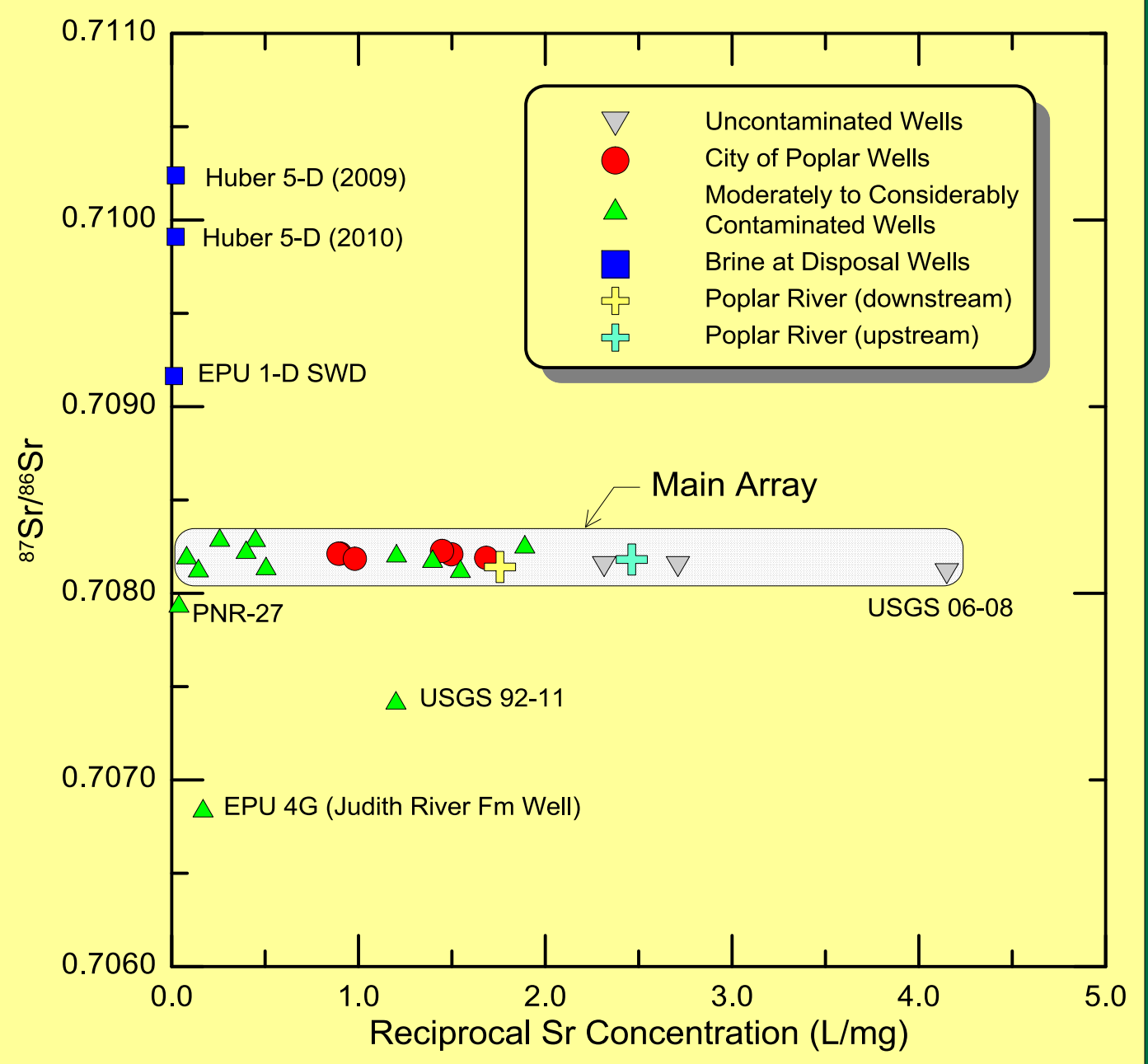

- Two sigma uncertainty in the ${ }^{87} \mathrm{Sr} /{ }^{86} \mathrm{Sr}$ ratio is 1.3E-5.

- Two sigma uncertainty of Sr concentration is 5 percent of the value or less. 


\section{${ }^{87} \mathrm{Sr} /{ }^{86} \mathrm{Sr}$ versus $1 / \mathrm{Sr}$ (Sr in mg/L)—(cont'd)}

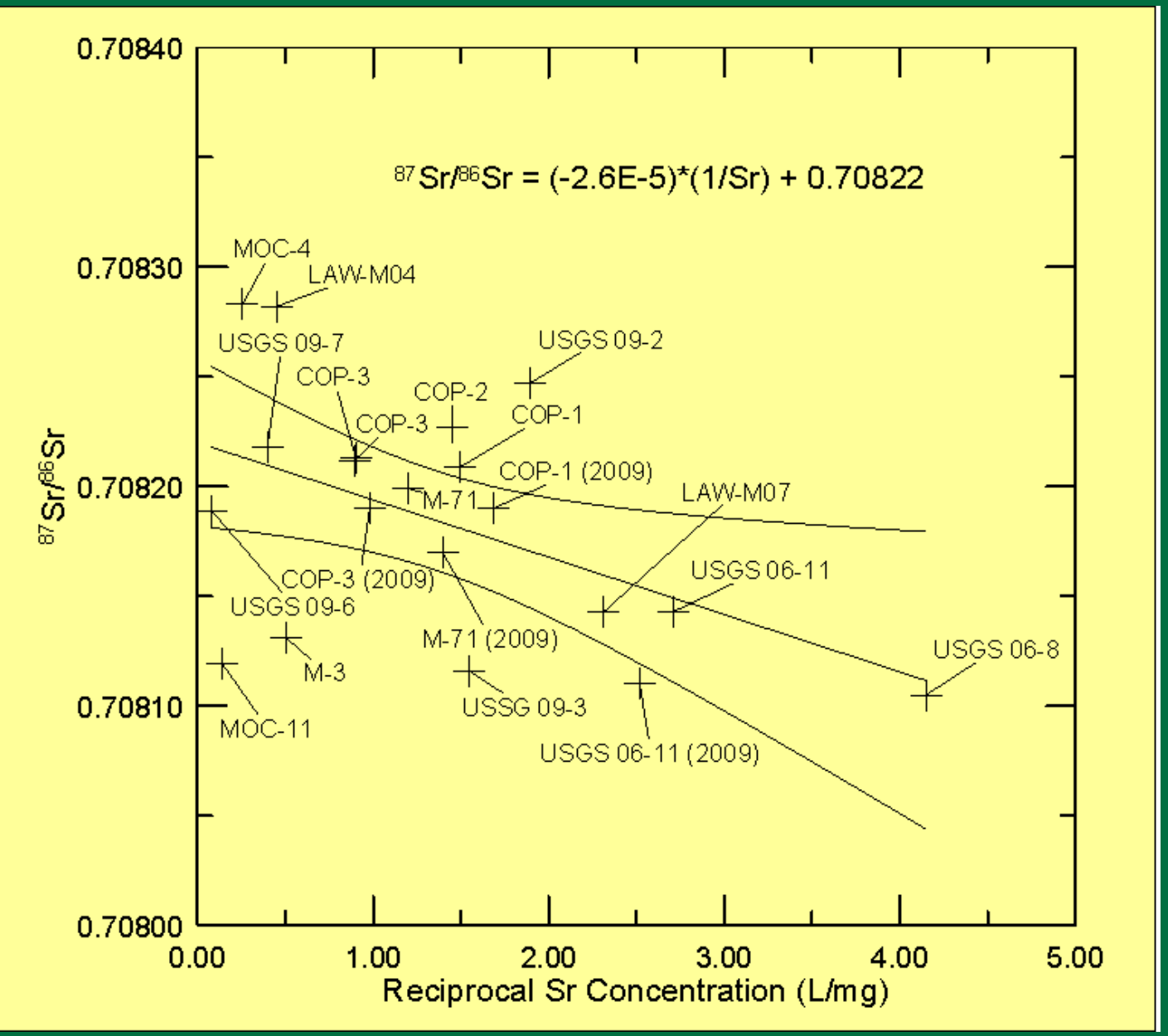

- Expansion of the Sr isotope axis of main array shows variability in ${ }^{87} \mathrm{Sr} /{ }^{87} \mathrm{Sr}$ and $\mathrm{Sr}$ concentrations

- The $Y$ intercept is likely the mean Sr isotope ratio of the contaminant that produced the main trend. 


\section{Spider Diagram for COP-1 and COP-3}

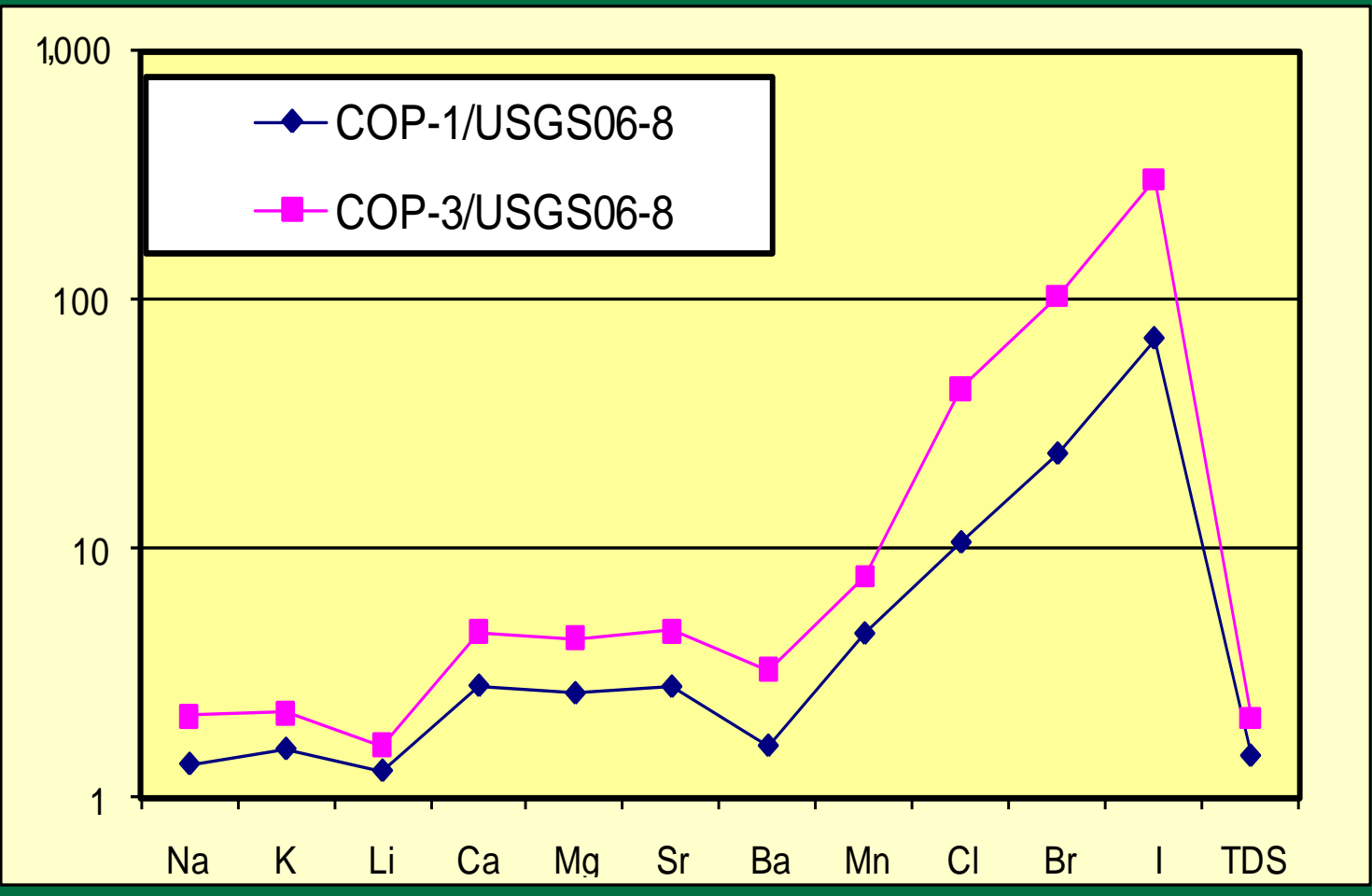

- Spider diagrams display ratios of concentrations of elements.

- COP-1, COP-2, and COP-3 are enriched in anions and cations that are abundant in brine.

- COP-2 is not shown because concentrations are similar to COP-1. 


\section{Conclusions}

- The main array is a contamination trend produced by mixing low $\mathrm{Sr}$ and low $\mathrm{Cl}$ waters $\left({ }^{87} \mathrm{Sr} /{ }^{86} \mathrm{Sr}\right.$ ratio of about 0.7081$)$ with brine $\left({ }^{87} \mathrm{Sr} /{ }^{86} \mathrm{Sr}\right.$ ratio averaging 0.70822$)$.

- Water samples from the COP wells plot along the main array and are enriched in constituents that are present in oil-field brines at high concentrations. 


\section{Conclusions (cont'd)}

- The brine that caused the main array is similar in ${ }^{87} \mathrm{Sr} /{ }^{86} \mathrm{Sr}$ to brines produced from the Madison Group elsewhere:

- 0.70802 at Goose Lake in northeast Montana (Peterman and others, 2010).

- $0.70811 \pm 0.00013$ at the Weyburn Field in southern Saskatchewan (Quattrocchi and others, 2006).

- The ${ }^{87} \mathrm{Sr} /{ }^{86} \mathrm{Sr}$ for water from the Judith River Fm indicates that upwelling is not likely to have affected the main array. 


\section{Supplemental Slides}

- $\mathrm{Ca}$ and $\mathrm{Cl}$ as a function of $1 / \mathrm{Sr}$

- Sample collection information and field measurements

- Major dissolved-ion concentrations

- Trace-metal concentrations

- Sr isotopes and Rb and Sr concentrations

- References 


\section{$1 / S r$ is an Index of Brine Contamination in the Main Array}
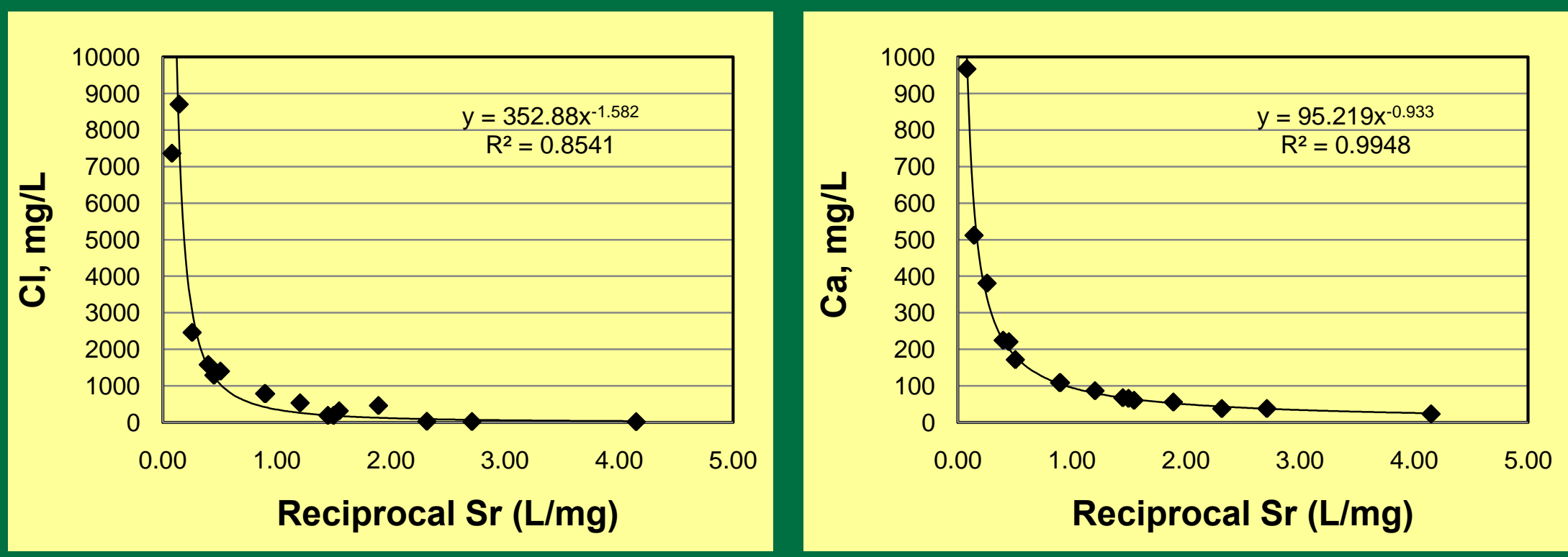


\section{Sample Collection Information and Field Measurements}

\begin{tabular}{|c|c|l|c|c|c|c|}
\hline Lab ID & Station ID & \multicolumn{1}{|c|}{ Station Name } & Sample Date Sample Time Field pH & Field SC \\
& & & & & & \\
\hline FPT-001 & 480849105102301 & LAW-M04 & $7 / 20 / 2010$ & 1730 & 7.3 & 4,680 \\
\hline FPT-002 & 481328105072702 & USGS 92-11 & $7 / 20 / 2010$ & 1930 & 7.9 & 6,360 \\
\hline FPT-003 & 480634105121501 & COP-3 & $7 / 21 / 2010$ & 1445 & 7.4 & 3,280 \\
\hline FPT-004 & 480634105121501 & COP-3 & $7 / 22 / 2010$ & 1440 & 7.4 & 3,280 \\
\hline FPT-005 & 480631105122101 & COP-2 & $7 / 21 / 2010$ & 1410 & 7.6 & 1,930 \\
\hline FPT-006 & 480630105121601 & COP-1 & $7 / 21 / 2010$ & 1330 & 7.6 & 1,900 \\
\hline FPT-007 & 480656105120001 & Poplar River PR-R-PR-042 & $7 / 21 / 2010$ & 1100 & 8.7 & 1,790 \\
\hline FPT-008 & 480855105103901 & LAW-M07 & $7 / 21 / 2010$ & 1715 & 7.7 & 1,210 \\
\hline FPT-009 & 481448105080001 & EPU 4-G & $7 / 22 / 2010$ & 0915 & -- & 36,400 \\
\hline FPT-010 & 481132105064801 & Huber 5-D & $7 / 22 / 2010$ & 0830 & -- & 122,400 \\
\hline FPT-011 & 481352105073401 & EPU 1-D SWD & $7 / 22 / 2010$ & 0900 & -- & 158,800 \\
\hline FPT-012 & 481143105090301 & M-3 & $7 / 22 / 2010$ & 1200 & 7.6 & 5,120 \\
\hline FPT-013 & 481146105074401 & MOC-11 & $7 / 22 / 2010$ & 1515 & 6.9 & 25,000 \\
\hline FPT-014 A & PNR-27 & PNR-27 & $7 / 22 / 2010$ & 1705 & 6.5 & 34,900 \\
\hline FPT-014 B & PNR-27 & PNR-27 & $7 / 22 / 2010$ & 1705 & 6.5 & 34,900 \\
\hline FPT-015 & 481635105051301 & Poplar River PR-R-PR-009 & $7 / 21 / 2010$ & 0930 & 8.9 & 1,480 \\
\hline FPT-016 & 481020105100201 & USGS 09-6 & $7 / 20 / 2010$ & 1600 & 6.9 & 20,100 \\
\hline FPT-017 & 480958105090301 & USGS 09-7 & $7 / 20 / 2010$ & 1430 & 7.3 & 5,510 \\
\hline FPT-018 & 481046105082301 & MOC-4 & $7 / 20 / 2010$ & 1130 & 7.2 & 8,748 \\
\hline FPT-019 & 481046105082301 & Field Blank coll. at MOC-4 & $7 / 20 / 2010$ & 1135 & -- & - \\
\hline FPT-020 & 480701105113201 & USGS 09-2 & $7 / 20 / 2010$ & 930 & 7.4 & 2,400 \\
\hline FPT-021 & 480720105105602 & USGS 06-8 & $7 / 19 / 2010$ & 1430 & 7.5 & 1,190 \\
\hline FPT-022 & 480729105112403 & USGS 06-11 & $7 / 19 / 2010$ & 1130 & 7.7 & 1,250 \\
\hline FPT-023 & 480809105094301 & USGS 09-3 & $7 / 19 / 2010$ & 1630 & 7.4 & 2,160 \\
\hline FPT-024 & 480814105102901 & M-71 & $7 / 19 / 2010$ & 1800 & 7.3 & 2,450 \\
\hline & & & & & \\
\hline & & & & & \\
\hline
\end{tabular}




\section{Major Dissolved lon Concentrations}

\begin{tabular}{|c|c|c|c|c|c|c|c|c|c|c|c|c|c|c|c|}
\hline Sample & & $\begin{array}{l}\text { Charge } \\
\text { Balance }\end{array}$ & $\begin{array}{c}\mathrm{Lab} \\
\mathrm{pH}\end{array}$ & $\begin{array}{c}\text { Lab } \\
\text { Specific } \\
\text { Conductance }\end{array}$ & $\mathrm{Na}$ & $\mathrm{Mg}$ & $\mathrm{K}$ & $\mathrm{Ca}$ & $\mathrm{F}$ & $\mathrm{Cl}$ & $\mathrm{HCO} 3$ & $\mathrm{SO}_{4}$ & $\mathrm{NO}_{3}$ & $\mathrm{Br}$ & I \\
\hline & Lab ID & percent & & $\mu \mathrm{S} / \mathrm{cm}$ & $\mathrm{mg} / \mathrm{L}$ & $\mathrm{mg} / \mathrm{L}$ & $\mathrm{mg} / \mathrm{L}$ & $\mathrm{mg} / \mathrm{L}$ & $\mathrm{mg} / \mathrm{L}$ & $\mathrm{mg} / \mathrm{L}$ & $\mathrm{mg} / \mathrm{L}$ & $\mathrm{mg} / \mathrm{L}$ & $\mathrm{mg} / \mathrm{L}$ & $\mathrm{mg} / \mathrm{L}$ & $\mathrm{mg} / \mathrm{L}$ \\
\hline \multicolumn{2}{|c|}{ Minimum Reporting Limit } & & & & 0.02 & 0.05 & 0.02 & 0.15 & 0.005 & 0.09 & 1.2 & 0.02 & 0.02 & 0.005 & 0.005 \\
\hline & & & & & & & & & & & & & & & \\
\hline LAW-M04 & FPT-001 & -0.2 & 7.4 & 4,640 & 556 & 153 & 12.6 & 221 & 0.18 & 1290 & 378 & 278 & 0.04 & 0.342 & 0.044 \\
\hline USGS 92-11 & FPT-002 & -1.1 & 8.1 & 6,330 & 1400 & 12.7 & 7.65 & 36.0 & 0.48 & 1730 & 614 & 309 & 0.08 & 0.643 & 0.057 \\
\hline $\mathrm{COP}-3$ & FPT-003 & 0.1 & 7.4 & 3,230 & 518 & 56.0 & 8.20 & 109 & 0.28 & 783 & 516 & 105 & $<0.02$ & 7.80 & 6.02 \\
\hline COP-3 & FPT-004 & -0.2 & 7.4 & 3,280 & 524 & 54.3 & 8.17 & 109 & 0.30 & 788 & 525 & 106 & $<0.02$ & 7.82 & 6.10 \\
\hline COP-2 & FPT-005 & 0.3 & 7.7 & 1,934 & 338 & 33.6 & 5.94 & 67.8 & 0.38 & 190 & 594 & 277 & 0.02 & 1.81 & 1.37 \\
\hline $\mathrm{COP}-1$ & FPT-006 & -0.1 & 7.6 & 1,926 & 337 & 32.6 & 5.87 & 66.0 & 0.38 & 189 & 600 & 271 & 0.11 & 1.81 & 1.38 \\
\hline Poplar River PR-R-PR-042 & FPT-007 & 0.8 & 8.7 & 1,779 & 331 & 50.3 & 9.08 & 28.9 & 0.42 & 120 & 642 & 287 & $<0.02$ & 0.128 & 0.019 \\
\hline LAW-M07 & FPT-008 & -0.5 & 7.8 & 1,211 & 223 & 22.4 & 4.57 & 38.1 & 0.39 & 28.1 & 540 & 194 & $<0.02$ & 0.082 & 0.017 \\
\hline EPU 4-G & FPT-009 & -0.3 & 6.5 & 18,490 & 4190 & 19.5 & 9.56 & 100 & 0.57 & 6610 & 214 & 2.67 & 2.9 & 66.2 & 50.3 \\
\hline Huber 5-D & FPT-010 & -1.2 & 7.0 & 116,400 & 32200 & 143 & 438 & 854 & 5.71 & 51800 & 274 & 1700 & 0.67 & 10.3 & 0.605 \\
\hline EPU 1-D SWD & FPT-011 & -1.4 & 7.0 & 150,000 & 42800 & 213 & 691 & 1460 & 5.05 & 70600 & 242 & 1350 & $<0.80$ & 23.0 & 1.78 \\
\hline M-3 & FPT-012 & -0.9 & 7.6 & 5,130 & 773 & 139 & 12.7 & 172 & 0.21 & 1400 & 543 & 315 & $<0.03$ & 0.494 & 0.079 \\
\hline MOC-11 & FPT-013 & -0.9 & 7.0 & 24,990 & 5180 & 274 & 26.0 & 512 & 0.21 & 8700 & 480 & 1250 & $<0.12$ & 17.9 & 9.40 \\
\hline PNR-27 & FPT-014 A & -0.8 & 6.7 & 34,900 & 5160 & 1320 & 61.9 & 1610 & 0.16 & 12900 & 722 & 2060 & 201 & 3.41 & 0.047 \\
\hline PNR-27 & FPT-014 B & -1.8 & 6.8 & 34,900 & 5070 & 1310 & 59.0 & 1610 & 0.16 & 13000 & 723 & 2070 & 201 & 3.46 & 0.046 \\
\hline Poplar River PR-R-PR-009 & FPT-015 & 0.7 & 8.8 & 1,499 & 297 & 40.0 & 8.33 & 19.0 & 0.45 & 11.8 & 669 & 279 & $<0.02$ & 0.079 & 0.012 \\
\hline USGS 09-6 & FPT-016 & -1.3 & 6.5 & 19,810 & 2310 & 778 & 33.2 & 967 & 0.10 & 7360 & 243 & 376 & $<0.10$ & 3.08 & 0.451 \\
\hline USGS 09-7 & FPT-017 & -1.0 & 7.4 & 5,480 & 718 & 174 & 12.9 & 225 & 0.16 & 1580 & 421 & 327 & $<0.03$ & 0.367 & 0.043 \\
\hline MOC-4 & FPT-018 & -0.6 & 7.2 & 8,690 & 1200 & 282 & 17.3 & 381 & 0.15 & 2460 & 443 & 928 & 0.05 & 1.33 & 0.272 \\
\hline Field Blank coll. at MOC-4 & FPT-019 & -27.0 & 6.5 & 1.4 & 0.04 & 0.092 & $<0.02$ & 0.43 & $<0.005$ & 0.023 & 3.2 & $<0.02$ & $<0.02$ & $<0.005$ & $<0.005$ \\
\hline USGS 09-2 & FPT-020 & -0.7 & 7.6 & 2,388 & 445 & 26.2 & 5.70 & 56.0 & 0.39 & 460 & 580 & 110 & $<0.02$ & 4.83 & 3.80 \\
\hline USGS 06-8 & FPT-021 & -0.1 & 7.7 & 1,166 & 248 & 12.5 & 3.76 & 23.6 & 0.43 & 18.0 & 558 & 165 & $<0.02$ & 0.076 & 0.020 \\
\hline USGS 06-11 & FPT-022 & 0.0 & 7.6 & 1,246 & 239 & 20.9 & 4.22 & 38.3 & 0.41 & 22.1 & 605 & 172 & 0.06 & 0.096 & 0.024 \\
\hline USGS 09-3 & FPT-023 & -0.8 & 7.7 & 2,147 & 374 & 34.1 & 6.02 & 60.7 & 0.26 & 315 & 487 & 272 & 3.7 & 0.150 & 0.034 \\
\hline M-71 & FPT-024 & -0.5 & 7.4 & 2,428 & 364 & 49.4 & 6.55 & 87.0 & 0.25 & 533 & 401 & 146 & $<0.02$ & 0.134 & 0.018 \\
\hline
\end{tabular}

\section{モUSGS}




\section{Trace-Metal Concentrations}

\begin{tabular}{|c|c|c|c|c|c|c|c|c|c|c|c|c|c|c|c|c|c|c|c|c|c|c|c|}
\hline Sample & & $\mathrm{Li}$ & $\mathrm{Be}$ & B & $\mathrm{Al}$ & $\mathrm{Cr}$ & $\mathrm{Mn}$ & Co & $\mathrm{Ni}$ & $\mathrm{Cu}$ & $\mathrm{Zn}$ & As & $\mathrm{Sr}$ & $\mathrm{Rb}$ & Mo & $\mathrm{Ag}$ & $\mathrm{Cd}$ & $\mathrm{Sb}$ & Cs & $\mathrm{Ba}$ & $\mathrm{Pb}$ & Th & $\mathrm{U}$ \\
\hline & Lab ID & $\mu \mathrm{g} / \mathrm{L}$ & $\mu \mathrm{g} / \mathrm{L}$ & $\mu \mathrm{g} / \mathrm{L}$ & $\mu \mathrm{g} / \mathrm{L}$ & $\mu \mathrm{g} / \mathrm{L}$ & $\mu \mathrm{g} / \mathrm{L}$ & $\mu \mathrm{g} / \mathrm{L}$ & $\mu \mathrm{g} / \mathrm{L}$ & $\mu \mathrm{g} / \mathrm{L}$ & $\mu \mathrm{g} / \mathrm{L}$ & $\mu \mathrm{g} / \mathrm{L}$ & $\mu \mathrm{g} / \mathrm{L}$ & $\mu \mathrm{g} / \mathrm{L}$ & $\mu \mathrm{g} / \mathrm{L}$ & $\mu \mathrm{g} / \mathrm{L}$ & $\mu \mathrm{g} / \mathrm{L}$ & $\mu \mathrm{g} / \mathrm{L}$ & $\mu \mathrm{g} / \mathrm{L}$ & $\mu \mathrm{g} / \mathrm{L}$ & $\mu \mathrm{g} / \mathrm{L}$ & $\mu \mathrm{g} / \mathrm{L}$ & $\mu \mathrm{g} / \mathrm{L}$ \\
\hline Minimum Reporting Limit & & 0.5 & 0.2 & 10 & 5 & 0.9 & 0.15 & 0.25 & 3 & 0.5 & 3 & 0.6 & 1 & 0.1 & 0.1 & 0.7 & 0.2 & 0.2 & 0.2 & 3 & 0.18 & 0.03 & 0.03 \\
\hline LAW-M04 & FPT-001 & 153 & $<2.0$ & 550 & $<50$ & $<9.0$ & 1180 & $<2.5$ & $<30$ & $<5.0$ & $<30$ & 8.3 & 2230 & $<1.0$ & 2.1 & $<7.0$ & $<2.0$ & $<2.0$ & $<2.0$ & 142 & $<1.8$ & $<0.30$ & 0.90 \\
\hline USGS 92-11 & FPT-002 & 183 & $<1.0$ & 756 & $<25$ & $<4.5$ & 143 & $<1.3$ & $<15$ & $<2.5$ & $<15$ & 3.5 & 833 & 0.8 & 5.5 & $<3.5$ & $<1.0$ & $<1.0$ & $<1.0$ & 42 & $<0.90$ & $<0.15$ & 1.95 \\
\hline COP-3 & FPT-003 & 127 & $<1.4$ & 609 & $<34$ & $<6.1$ & 890 & $<1.7$ & $<20$ & $<3.4$ & $<20$ & 8.5 & 1110 & 2.7 & 8.0 & $<4.7$ & $<1.4$ & $<1.4$ & $<1.4$ & 89 & $<1.2$ & $<0.20$ & 1.35 \\
\hline COP-3 & FPT-004 & 122 & $<1.4$ & 587 & $<34$ & $<6.1$ & 867 & $<1.7$ & $<20$ & $<3.4$ & $<20$ & 8.5 & 1120 & 2.7 & 8.6 & $<4.7$ & $<1.4$ & $<1.4$ & $<1.4$ & 91 & $<1.2$ & $<0.20$ & 1.35 \\
\hline COP-2 & FPT-005 & 98 & $<0.8$ & 623 & $<20$ & 4.3 & 526 & $<1.0$ & $<12$ & $<2.0$ & $<12$ & 6.5 & 691 & 2.1 & 11 & $<2.8$ & $<0.8$ & $<0.8$ & $<0.8$ & 44 & $<0.73$ & $<0.12$ & 1.24 \\
\hline COP-1 & FPT-006 & 97 & $<0.8$ & 633 & $<20$ & 4.1 & 507 & $<1.0$ & $<12$ & $<2.0$ & $<12$ & 8.3 & 668 & 2.0 & 11 & $<2.8$ & $<0.8$ & $<0.8$ & $<0.8$ & 45 & $<0.73$ & $<0.12$ & 1.17 \\
\hline Poplar River PR-R-PR-042 & FPT-007 & 106 & $<0.8$ & 1100 & $<20$ & 4.7 & 3.7 & 1.1 & $<12$ & $<2.0$ & $<12$ & 4.6 & 569 & 1.6 & 2.8 & $<2.8$ & $<0.8$ & $<0.8$ & $<0.8$ & 65 & $<0.73$ & $<0.12$ & 2.07 \\
\hline LAW-M07 & FPT-008 & 75 & $<0.5$ & 604 & $<13$ & 4.1 & 147 & $<0.6$ & $<7.5$ & $<1.3$ & $<7.5$ & 10 & 432 & 0.6 & 5.7 & $<1.8$ & $<0.5$ & $<0.5$ & $<0.5$ & 36 & $<0.45$ & $<0.08$ & 0.71 \\
\hline EPU 4-G & FPT-009 & 411 & $<2.0$ & 4450 & $<50$ & $<9.0$ & 85 & $<2.5$ & $<30$ & $<5.0$ & 396 & 17 & 5960 & 8.4 & $<1.0$ & $<7.0$ & $<2.0$ & $<2.0$ & $<2.0$ & 6930 & 2.36 & $<0.30$ & $<0.30$ \\
\hline Huber 5-D & FPT-010 & 4040 & $<4.9$ & 14300 & $<123$ & $<22$ & 130 & $<6.1$ & $<74$ & $<12$ & $<74$ & 73 & 47300 & 974 & $<2.5$ & $<17$ & $<4.9$ & $<4.9$ & 82 & 564 & $<4.4$ & $<0.74$ & $<0.74$ \\
\hline EPU 1-D SWD & FPT-011 & 6000 & $<5.8$ & 32200 & $<146$ & $<26$ & 62 & $<7.3$ & $<87$ & $<15$ & $<87$ & 92 & 83000 & 1580 & $<2.9$ & $<20$ & $<5.8$ & $<5.8$ & 139 & 1120 & $<5.2$ & $<0.87$ & $<0.87$ \\
\hline M-3 & FPT-012 & 159 & $<2.0$ & 741 & $<50$ & $<9.0$ & 400 & $<2.5$ & $<30$ & $<5.0$ & $<30$ & 10 & 1980 & 1.2 & 2.7 & $<7.0$ & $<2.0$ & $<2.0$ & $<2.0$ & 104 & $<1.8$ & $<0.30$ & 1.08 \\
\hline MOC-11 & FPT-013 & 736 & $<2.0$ & 676 & $<50$ & $<9.0$ & 1240 & 3.0 & $<30$ & $<5.0$ & $<30$ & 29 & 6990 & 2.6 & 2.9 & $<7.0$ & $<2.0$ & $<2.0$ & $<2.0$ & 49 & $<1.8$ & $<0.30$ & 2.64 \\
\hline PNR-27 & FPT-014 A & 973 & $<2.0$ & 502 & $<50$ & $<9.0$ & 107 & $<2.5$ & $<30$ & 7.4 & $<30$ & 29 & 26000 & 6.8 & $<1.0$ & $<7.0$ & $<2.0$ & $<2.0$ & $<2.0$ & 52 & $<1.8$ & $<0.30$ & 76.5 \\
\hline PNR-27 & FPT-014 B & 958 & $<2.0$ & 494 & $<50$ & $<9.0$ & 105 & $<2.5$ & $<30$ & 7.4 & $<30$ & 31 & 26000 & 6.9 & $<1.0$ & $<7.0$ & $<2.0$ & $<2.0$ & $<2.0$ & 52 & $<1.8$ & $<0.30$ & 77.4 \\
\hline Poplar River PR-R-PR-009 & FPT-015 & 99 & $<0.7$ & 1070 & 23 & 4.9 & 2.7 & 1.3 & $<10$ & $<1.7$ & $<10$ & 4.6 & 406 & 1.5 & 2.7 & $<2.4$ & $<0.7$ & $<0.7$ & $<0.7$ & 47 & $<0.60$ & $<0.10$ & 1.81 \\
\hline USGS 09-6 & FPT-016 & 369 & $<2.0$ & 598 & $<50$ & $<9.0$ & 5150 & $<2.5$ & $<30$ & $<5.0$ & $<30$ & 24 & 12500 & 3.4 & $<1.0$ & $<7.0$ & $<2.0$ & $<2.0$ & $<2.0$ & 321 & $<1.8$ & $<0.30$ & 1.07 \\
\hline USGS 09-7 & FPT-017 & 172 & $<2.0$ & 662 & $<50$ & $<9.1$ & 1030 & $<2.5$ & $<30$ & $<5.0$ & $<30$ & 9.4 & 2510 & 1.1 & 1.5 & $<7.0$ & $<2.0$ & $<2.0$ & $<2.0$ & 159 & $<1.8$ & $<0.30$ & 0.68 \\
\hline MOC-4 & FPT-018 & 225 & $<1.0$ & 632 & $<25$ & $<4.5$ & 2290 & $<1.3$ & $<15$ & $<2.5$ & $<15$ & 11 & 3890 & 1.6 & 1.6 & $<3.5$ & $<1.0$ & $<1.0$ & $<1.0$ & 60 & $<0.91$ & $<0.15$ & 1.95 \\
\hline Field Blank coll. at MOC-4 & FPT-019 & $<0.5$ & $<0.2$ & $<10$ & $<5$ & $<0.9$ & 0.9 & $<0.3$ & $<3$ & $<0.5$ & $<3$ & $<0.6$ & $<1.0$ & $<0.1$ & $<0.1$ & $<0.7$ & $<0.2$ & $<0.2$ & $<0.2$ & $<3$ & $<0.18$ & $<0.03$ & $<0.03$ \\
\hline USGS 09-2 & FPT-020 & 83 & $<1.0$ & 545 & $<25$ & $<4.6$ & 206 & $<1.3$ & $<15$ & $<2.5$ & $<15$ & 8.3 & 529 & 1.3 & 10 & $<3.6$ & $<1.0$ & $<1.0$ & $<1.0$ & 49 & $<0.91$ & $<0.15$ & 1.66 \\
\hline USGS 06-8 & FPT-021 & 76 & $<0.5$ & 552 & $<13$ & 4.3 & 112 & $<0.6$ & $<7.5$ & $<1.3$ & $<7.5$ & 8.2 & 241 & 0.9 & 11 & $<1.8$ & $<0.5$ & $<0.5$ & $<0.5$ & 28 & $<0.45$ & $<0.08$ & 0.76 \\
\hline USGS 06-11 & FPT-022 & 75 & $<0.5$ & 522 & $<13$ & 4.7 & 269 & $<0.6$ & $<7.6$ & $<1.3$ & $<7.6$ & 9.3 & 369 & 1.0 & 11 & $<1.8$ & $<0.5$ & $<0.5$ & $<0.5$ & 31 & $<0.45$ & $<0.08$ & 1.90 \\
\hline USGS 09-3 & FPT-023 & 101 & $<1.0$ & 631 & $<25$ & $<4.5$ & 386 & $<1.3$ & $<15$ & $<2.5$ & $<15$ & 11 & 647 & 0.8 & 3.9 & $<3.5$ & $<1.0$ & $<1.0$ & $<1.0$ & 112 & $<0.91$ & $<0.15$ & 1.10 \\
\hline M-71 & FPT-024 & 115 & $<1.0$ & 533 & $<25$ & $<4.6$ & 725 & $<1.3$ & $<15$ & $<2.5$ & $<15$ & 8.2 & 831 & 0.7 & 3.8 & $<3.6$ & $<1.0$ & $<1.0$ & $<1.0$ & 73 & $<0.92$ & $<0.15$ & 0.79 \\
\hline
\end{tabular}




\begin{tabular}{|c|c|c|c|c|}
\hline \multirow[t]{2}{*}{ Sample } & & $\mathrm{Sr}$ & $\mathrm{Rb}$ & ${ }^{87} \mathrm{Sr} /{ }^{86} \mathrm{Sr}$ \\
\hline & Lab ID & $\mu g / L$ & $\mu \mathrm{g} / \mathrm{L}$ & Atom ratio \\
\hline \multicolumn{2}{|l|}{ Minimum Reporting Limit } & 1 & 0.1 & NA \\
\hline $\mathrm{J} A \mathrm{~W}$ & EDT $\Omega$ & 2020 & $<10$ & 070828 \\
\hline USGS 92-11 & FPT-002 & 833 & 0.8 & 0.70741 \\
\hline USGS 92-11 (Sr dup) & FPT-002 & & & 0.70741 \\
\hline COP-3 & FPT-003 & 1,110 & 2.7 & 0.70821 \\
\hline $\mathrm{COP}-3$ & FPT-004 & 1,120 & 2.7 & 0.70821 \\
\hline COP-3 (2009) & & 1,020 & $<5$ & 0.70819 \\
\hline COP-2 & FPT-005 & 691 & 2.1 & 0.70823 \\
\hline COP-1 & FPT-006 & 668 & 2.0 & 0.70821 \\
\hline COP-1 (2009) & & 594 & 2.3 & 0.70819 \\
\hline Poplar River PR-R-PR-042 & FPT-007 & 569 & 1.6 & 0.70814 \\
\hline LAW-M07 & FPT-008 & 432 & 0.6 & 0.70814 \\
\hline EPU 4-G & FPT-009 & 5,960 & 8.4 & 0.70683 \\
\hline Huber 5-D & FPT-010 & 47,300 & 974 & 0.70991 \\
\hline Huber 5-D (2009) & & 45,700 & 1080 & 0.71024 \\
\hline EPU 1-D SWD & FPT-011 & 83,000 & 1580 & 0.70916 \\
\hline M-3 & FPT-012 & 1,980 & 1.2 & 0.70813 \\
\hline MOC-11 & FPT-013 & 6,990 & 2.6 & 0.70812 \\
\hline PNR-27 & FPT-014 A & 26,000 & 6.8 & 0.70793 \\
\hline PNR-27 (Sr dup) & FPT-014 A & & & 0.70795 \\
\hline PNR-27 & FPT-014 B & 26,000 & 6.9 & --- \\
\hline Poplar River PR-R-PR-009 & FPT-015 & 406 & 1.5 & 0.70818 \\
\hline USGS 09-6 & FPT-016 & 12,500 & 3.4 & 0.70819 \\
\hline USGS 09-7 & FPT-017 & 2,510 & 1.1 & 0.70822 \\
\hline MOC-4 & FPT-018 & 3,890 & 1.6 & 0.70828 \\
\hline Field Blank coll. at MOC-4 & FPT-019 & $<1.0$ & $<0.1$ & --- \\
\hline USGS 09-2 & FPT-020 & 529 & 1.3 & 0.70825 \\
\hline USGS 06-8 & FPT-021 & 241 & 0.9 & 0.70811 \\
\hline USGS 06-11 & FPT-022 & 369 & 1.0 & 0.70814 \\
\hline USGS 06-11 (2009) & & 397 & 1.2 & 0.70811 \\
\hline USGS 09-3 & FPT-023 & 647 & 0.8 & 0.70812 \\
\hline M-71 & FPT-024 & 831 & 0.7 & 0.70820 \\
\hline M-71 (2009) & & 716 & $<5$ & 0.70817 \\
\hline
\end{tabular}

\section{Sr lsotopes and $\mathbf{R b}$ and $\mathrm{Sr}$ Concentrations}




\section{References}

- Faure, Gunter, 1998, Principles and applications of geochemistry: Prentice-Hall, Upper Saddle River, New Jersey, $2^{\text {nd }}$ ed., p. 335-337.

- Levings, G.W., 1984, Reconnaissance evaluation of contamination in the alluvial aquifer in the East Poplar oil field, Roosevelt County, Montana: U.S. Geological Survey Water-Resources Investigations Report 84-4174, 29 p.

- Peterman, Z.P., Thamke, J.N., and Futa, K., 2010, Use of strontium isotopes to detect produced-water contamination in surface water and groundwater in the Williston Basin, northeastern Montana [abs.]: GeoCanada 2010 Conference, Calgary, Alberta, May 10-14, 2010.

- Quattrocchi, F., Barbieri, M., Bencini, R., Cinti, D., Durocher, K., Galli, G., Pizzino, I., Shevalier, M., and Voltattorni, N., 2006, Strontium isotope $\left({ }^{87} \mathrm{Sr} / 86^{\mathrm{Sr}}\right)$ chemistry in produced oil field waters: The IEA $\mathrm{CO}_{2}$ Monitoring and Storage Project: Advances in the Geological Storage of Carbon Dioxide, Springer, The Netherlands.

- S.S. Papadopulos \& Associates, Inc., 2008, Phase 2 report, Hydrogeologic assessment of chloride-migration potential in the vicinity of Poplar, Montana: Boulder, Colo., prepared for Fort Peck Assiniboine \& Sioux Tribes, [variously paged].

- Thamke, J.N., and Craigg, S.D., 1997, Saline-water contamination in Quaternary deposits and the Poplar River, East Poplar oil field, northeastern Montana: U.S. Geological Survey Water-Resources Investigations Report 97-4000, 37 p.

- Thamke, J.N., and Midtlyng, K.S., 2003, Ground-water quality for two areas in the Fort Peck Indian Reservation, northeastern Montana: U.S. Geological Survey Water-Resources Investigations Report 2003-4214, 54 p.

- U.S. Environmental Protection Agency, 2010, Electronic Code of Federal Regulations, secondary maximum contaminant levels, accessed online at: httpil/ecfirgpoaccess_gov/cgi/t/text/text-

idx?c=ecfirsid=480724f02292bea059f258c5647f2abfirgn=div5\& view=text \&node=40:22,0.1.1.5\&idno $=40: 40=22,0.1 .1,5.0 .39 .3$

- Wilson, Marjorie, 1989, Igneous petrogenesis-A global tectonic approach: London, Unwin Hyman, p. 19-21. 
